\title{
Nutritional Rehabilitative Treatment in a Residential Centre for Eating Disorders: A Case Series Report
}

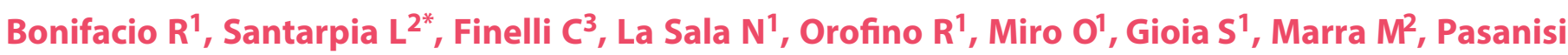 \\ $F^{2}$, Contaldo $F^{2}$ and Trabace $\mathbf{R}^{1}$ \\ ${ }^{1}$ Center for the Treatment of Eating Disorders, Chiaromonte, Potenza, Italy \\ ${ }^{2}$ Department of Clinical Medicine and Surgery, Center for Obesity and Eating Disorders (CISRODCA), Federico II University, Naples, Italy \\ ${ }^{3}$ Department of Medicine, San Giovanni Hospital, Chiaromonte, Potenza, Italy
}

*Corresponding author: Santarpia L, Department of Clinical Medicine and Surgery, Center for Obesity and Eating Disorders (CISRODCA), Federico II University, Naples, Italy, Tel: 0039081746 2333; E-mail: lidia.santarpia@unina.it

Received date: June 16, 2016; Accepted date: July 04, 2016; Published date: July 11, 2016

Citation: Bonifacio R, Santarpia L, Finelli C, La Sala N, Orofino R, et al. (2016) Nutritional Rehabilitative Treatment in a Residential Centre for Eating Disorders: A Case Series Report. J Obes Eat Disord 2: 2. doi: http://dx.doi.org/10.4172/2471-8203.100018

Copyright: (c 2016, Bonifacio R, et al. This is an open-access article distributed under the terms of the Creative Commons Attribution License, which permits unrestricted use, distribution, and reproduction in any medium, provided the original author and source are credited.

\section{Abstract}

Purpose: Patients with Eating Disorders (ED) could benefit of temporary leaving from their family and social environment, hosting in specialized residential centers.

Methods: Fifty-one female patients ( $23.5 \pm 6.1$ years), 22 with Anorexia Nervosa (AN), 14 with Bulimia Nervosa (BN), 9 with Binge Eating Disorders (BED) and 6 with nototherwise specified eating disorders (EDNOS) were followed in a residential regimen for at least 3 months.

Anthropometric, clinical, biochemical and body composition parameters were evaluated at entry, at 1, 3 and 5 months, while residents and 6 months after discharge. A multidisciplinary team, including specialized medical doctors, psychotherapists and dieticians was involved in the treatment.

Results: A significant $(p<0.001)$ and progressive weight gain in $22 \mathrm{AN}$ patients, both at the end of the residential period and at 6 month follow-up was observed, with no signs of refeeding syndrome. Resting Energy Expenditure (REE) progressively increased, reaching significance $(p<0.02)$ at 5 month treatment. Menses spontaneously recovered in 12 patients, after 17 (5-48) months amenorrhea. In $14 \mathrm{BN}$ patients, binge eating and purging episodes were eliminated. Nine BED patients gradually lost weight $(-14.5 \pm 3.2 \mathrm{~kg})$ and in particular Fat Mass $(F M)$, with a significant $(p<0.05)$ improvement in blood lipids and serum transaminases. No significant changes were observed in the 6 EDNOS patients, possibly due to the low number and wide variability of patients gathered in this disease definition.

Conclusion: These observations suggest the safety, with the improvement of several clinical and nutritional parameters, of a multi-disciplinary and integrated approach carried out in a residential setting, in particular for AN patients.

Keywords: Eating disorders; Nutritional rehabilitation; Residential treatment

\section{Introduction}

Eating Disorders (EDs) incidence is constantly increasing and spreading among female (and to a lesser extent male) adolescents from all social and cultural environments [1-4]. On the other hand, adolescence nowadays comprises a wider age range of individuals compared to the past, also due to the continuous and fast cultural and socio-economic changes [5-7].

For the treatment of EDs a multi-disciplinary approach is suggested. Basically, the therapeutic team should include physicians specialized in clinical nutrition, psychiatrists, psychologists, dieticians and other supportive rehabilitative professionals [8-11]. Generally, these disorders are treated on an outpatient basis; only in selected/severe cases hospital admission is required [12-16].

For the treatment of the disease, patients can take advantage from the temporary leaving from the family/social environment often described to cause and sustain the dysfunctional behaviour [17-21]. This kind of intervention is obtained by hosting ED patients in specialized Centers, usually for a period of time longer than 90 days. However, in the literature, at least at our knowledge, there is little description of the short and long term outcome (relapse, complications, effectiveness) of this therapeutic approach.

\section{Aim of the Study}

To evaluate in a group of ED patients (Anorexia Nervosa (AN), Bulimia Nervosa (BN), Binge Eating Disorders (BED), Not 
Otherwise Specified Eating Disorders (EDNOS) tolerability, safety and clinical nutritional effects of a 3-5 months multidisciplinary residential rehabilitative treatment

\section{Methods}

Seventy-nine patients were admitted at the Center for the Treatment of Eating Disorders "G. Gioia" in Chiaromonte (PZ), Italy, from 2010 to 2012. Permanence in the residential Center lasted at least 3 months.

Four male patients and 24 patients (age 24, $1 \pm 7$ years) who did not attend the follow-up (FU) visit were not included in the study. Therefore, 51 female patients, suffering from ED (AN, $B N, B E D, E D N O S$ ) and attending the scheduled follow up visit, were studied. Social and demographic data as well as clinical and biochemical characteristics, anthropometry, body composition, Resting Energy Expenditure (REE) and some psychometric parameters (data not shown) at different steps of the treatment were evaluated.

Before admission at the rehabilitative residential Center, patients were evaluated by a psychiatrist. The diagnosis of ED was made according to DSM-IV criteria [22], because the data were collected from 2010-2012, and lately confirmed according to DSM-V criteria [23]. Patients with major psychiatric disorders, acute diseases and/or Body Mass Index (BMI=weight/squared height ) $<13 \mathrm{Kg} / \mathrm{m}^{2}$ were not admitted to the residential therapeutic program.

Socio-demographic characteristics and disease history were collected at entry. Anthropometry (weight, height, BMI), clinical (Blood Pressure, Heart Rate, ECG), biochemical and body composition parameters were registered at entry and at 1-3 and $5^{\text {th }}$ month of the residential period and 6 months after discharge.

Body composition was evaluated by BIA (Human II Plus DS Medica) and Resting Energy Expenditure (REE) by indirect calorimetry (VMax Sensor Medics) in standardized conditions: after an overnight fasting in a quite, thermo-neutral $\left(22^{\circ} \mathrm{C}\right.$ temperature) environment. Nutritional intervention was prescribed by the nutritionist according to patient's energy requirements measured by indirect calorimetry and to the diet history collected at entry. Diet energy content variations during the residential period were allowed according to REE measurements. The prescribed diet was prepared following the Mediterranean Diet style and the National Guidelines for a healthy diet. At entry and at discharge, liver ultrasonography (Esaote Mpx Biomedica) was performed to evaluate the presence and grade of steatosis. Both a convex $(3.5-\mathrm{MHz})$ and a linear high frequency $(7-13 \mathrm{MHz})$ probes were used. The presence of liver steatosis was classified according to Saverymuttu et al. [24] criteria with a semi-quantitative method on a 0-3 scale: (0: normal liver; 1 : slight steatosis; 2 : moderate steatosis; 3 : severe steatosis).

At entry, after 1-3-5 months of residential treatment and at the following FU routine haemato-biochemical parameters were evaluated: serum glucose, total cholesterol, HDL and LDL cholesterol, triglycerides, urea, electrolytes ( $\mathrm{Na}, \mathrm{K}, \mathrm{Cl}, \mathrm{Ca}, \mathrm{P}$,
$\mathrm{Mg}$ ), blood count, transaminases, GGT, CK, fibrinogen, total proteins and albumin. Serum uric acid, Sedimentation Rate $(\mathrm{SR})$, iron and ferritin, amylase and thyroid hormones within the normal range at admission, were not measured again.

Collected data were analysed with SPSS 15.0 software for statistical analysis. Parameters measured at specific time points were compared with values at admission by using t-test for paired data. Significance was considered for $p$ values $<0.05$.

\section{Therapeutic protocol}

Patients were supervised by professional educators: daily activities occurred in defined areas of the residential structure and each outdoor activity was accurately planned. Diet was prescribed by a clinical nutritionist, calculated a discussed with the patient by the dietician, according to the individual needs measured by indirect calorimetry, BIA and to the dietetic history collected at entry, with adaptations and integrations, when necessary. Meals were prepared in the hospital dining hall, and offered in personalized trays. Five meals a day were scheduled with the assistance and supervision of a dietician and a professional educator, in a dedicated room furnished with tables each with 4 seats. Rules of conduct, such as: no comments on food, no break into pieces, no refuse, no ruminate/voracity, etc were warned. Hygienic services were locked for 2 hours after each meal, to discourage compensatory behaviors. According to the therapeutic protocol, no foods or drugs could be brought in without previous approval. Daily nurse assistance and weekly clinical monitoring (Blood Pressure (BP), Heart Rate (HR), review of symptoms) by the physicians were scheduled.

Weekly psychotherapeutic and nutritional, either individual (on average lasting 20-30 min) or group meetings (on average lasting $90 \mathrm{~min}$ ), were scheduled. Gynecological counseling at entry was offered, when necessary. Integrative activities with dedicated operators (music-therapy, art-therapy, communication groups, pet-therapy with horses and donkeys, shiatsu and physiotherapy, on average corresponding to 10-15 h/weekly activity, subdividing patients in 2-3 groups) were planned. For overweight/obese patients 30-40 minutes/day aerobic (tapis-roulant, fast walking) physical activity was scheduled. Therapeutic rehabilitative program was individually scheduled and monitored during weekly team meetings.

On average, 6 weeks after admission, patients could eat some snacks and meals without supervision, out of the residential environment; parents presence was eventually accorded after team discussion. At about 8 weeks after admission, patients could return home to spend their weekends. Patients were prepared for this experience, among others, participating to a cooking laboratory in the hospital dining hall with the supervision of a dietician. In this experience, patients progressively learnt how to manage their nutritional therapy, preparing, weighting and flavouring foods.

Fortnightly family meetings with psychologist and clinical nutritionist were scheduled to discuss difficulties and strategies to face individual and relational problems; monthly 
meetings of all patients' families were organized with the presence of social assistants for reciprocal help.

Table 1: General characteristics of 51 patients with Eating Disorders, divided according to their diseases.

\begin{tabular}{|c|c|c|c|c|c|c|c|c|c|c|}
\hline Eating Disorder & \multicolumn{2}{|c|}{$\begin{array}{l}\text { Anorexia } \\
\text { Nervosa }\end{array}$} & \multicolumn{2}{|c|}{ Bulimia Nervosa } & \multicolumn{2}{|c|}{$\begin{array}{l}\text { Binge } \quad \text { Eating } \\
\text { Disorders }\end{array}$} & \multicolumn{2}{|c|}{ EDNOS } & \multicolumn{2}{|c|}{ TOTAL } \\
\hline $\begin{array}{l}\text { Number of patients, } \\
(\%)\end{array}$ & \multicolumn{2}{|c|}{$22,(-43.2)$} & \multicolumn{2}{|c|}{$14,(-27.5)$} & \multicolumn{2}{|c|}{$9,(-17.6)$} & \multicolumn{2}{|c|}{$6,(-11.7)$} & \multicolumn{2}{|c|}{$51,(-100)$} \\
\hline Age (years), (range) & \multicolumn{2}{|c|}{$\begin{array}{l}23.1 \pm 5.3 \\
(13-32)\end{array}$} & \multicolumn{2}{|c|}{$\begin{array}{l}24 \pm 7.7 \\
(14-39)\end{array}$} & \multicolumn{2}{|c|}{$24 \pm 7.4,(16-40)$} & \multicolumn{2}{|c|}{$\begin{array}{l}23.3 \pm 4.2 \\
(16-29)\end{array}$} & \multicolumn{2}{|c|}{$\begin{array}{l}23.5 \pm 6.1 \\
(13-40)\end{array}$} \\
\hline Age of onset (years), (range) & \multicolumn{2}{|c|}{$\begin{array}{l}17 \pm 4.1 \\
(10-28)\end{array}$} & \multicolumn{2}{|c|}{$\begin{array}{l}15.5 \pm 2.3 \\
(13-23)\end{array}$} & \multicolumn{2}{|c|}{$\begin{array}{l}15.4 \pm 4.6 \\
(10-24)\end{array}$} & \multicolumn{2}{|c|}{$\begin{array}{l}16.7 \pm 3 \\
(14-21)\end{array}$} & \multicolumn{2}{|c|}{$\begin{array}{l}16.3 \pm 3.7 \\
(10-28)\end{array}$} \\
\hline $\begin{array}{l}\text { Disease length (years), } \\
\text { (range) }\end{array}$ & \multicolumn{2}{|c|}{$\begin{array}{l}5.9 \pm 4.3 \\
(1-17)\end{array}$} & \multicolumn{2}{|c|}{$\begin{array}{l}8.5 \pm 7.9 \\
(1-24)\end{array}$} & \multicolumn{2}{|c|}{$\begin{array}{l}8.5 \pm 5.6 \\
(2-17)\end{array}$} & \multicolumn{2}{|c|}{$\begin{array}{l}6.7 \pm 4.6 \\
(1-14)\end{array}$} & \multicolumn{2}{|c|}{$\begin{array}{l}7.2 \pm 5.7 \\
(1-24)\end{array}$} \\
\hline Residential period, (months) & \multicolumn{2}{|c|}{$\begin{array}{l}4.9 \pm 0.7 \\
(3-5)\end{array}$} & \multicolumn{2}{|c|}{$\begin{array}{l}5.0 \pm 0.0 \\
(5)\end{array}$} & \multicolumn{2}{|c|}{$\begin{array}{l}5.0 \pm 0.0 \\
(5)\end{array}$} & \multicolumn{2}{|c|}{$\begin{array}{l}4.7 \pm 0.8 \\
(3-5)\end{array}$} & \multicolumn{2}{|c|}{$\begin{array}{l}4.9 \pm 0.5 \\
(3-5)\end{array}$} \\
\hline Region of Provenience & $\mathbf{n}$ & $\%$ & $\mathbf{n}$ & $\%$ & $\mathbf{n}$ & $\%$ & $\mathbf{n}$ & $\%$ & $\mathbf{n}$ & $\%$ \\
\hline Southern Italy & 19 & 86.4 & & & 9 & 100 & 6 & 100 & 48 & 94 \\
\hline Basilicata & 4 & 18.2 & 8 & 57.2 & 3 & 33.3 & 2 & 33.3 & 17 & 33.3 \\
\hline Campania & 9 & 40.9 & 2 & 14.3 & 0 & 0 & 1 & 16.7 & 12 & 23.5 \\
\hline Puglia & 3 & 13.6 & 0 & 0 & 3 & 33.3 & 1 & 16.7 & 7 & 13.7 \\
\hline Calabria & 2 & 9.2 & 3 & 21.4 & 3 & 33.3 & 2 & 33.3 & 10 & 19.6 \\
\hline Sicilia & 1 & 4.5 & 1 & 7.1 & 0 & 0 & 0 & 0 & 2 & 3.9 \\
\hline North- Centre Italy & 3 & 13.6 & 0 & 0 & 0 & 0 & 0 & 0 & 3 & 6.0 \\
\hline School Degree & $\mathbf{n}$ & $\%$ & $\mathbf{n}$ & $\%$ & $\mathbf{n}$ & $\%$ & $\mathbf{n}$ & $\%$ & $\mathbf{n}$ & $\%$ \\
\hline Elementary school & 1 & 4.5 & 0 & 0 & 0 & 0 & 0 & 0 & 1 & 2.0 \\
\hline Junior high school & 4 & 18.2 & 2 & 14.3 & 1 & 11.1 & 0 & 11.1 & 7 & 13.7 \\
\hline High school & 13 & 59.1 & 12 & 85.7 & 7 & 77.8 & 4 & 6.7 & 36 & 70.6 \\
\hline Degree & 4 & 18.2 & 0 & & 1 & 11.1 & 2 & 3.3 & 7 & 13.7 \\
\hline Head of the household work & $\mathbf{n}$ & $\%$ & $\mathbf{n}$ & $\%$ & $\mathbf{n}$ & $\%$ & $\mathbf{n}$ & $\%$ & $\mathbf{n}$ & $\%$ \\
\hline $\begin{array}{l}\text { Retail merchant/ } \\
\text { employee/ worker }\end{array}$ & 11 & 50.0 & 9 & 64.3 & 7 & 77.8 & 4 & 66.7 & 31 & 60.8 \\
\hline $\begin{array}{l}\text { Self-employed/ } \\
\text { manager/director }\end{array}$ & 8 & 36.4 & 3 & 21.4 & 2 & 22.2 & 0 & 0 & 13 & 25.5 \\
\hline Retired/died & 3 & 13.6 & 2 & 14.3 & 0 & 0 & 1 & 16.7 & 6 & 11.7 \\
\hline Unemployed & 0 & 0 & 0 & 0 & 0 & 0 & 1 & 16.7 & 1 & 2.0 \\
\hline Profession & $\mathbf{n}$ & $\%$ & $\mathrm{n}$ & $\%$ & $\mathbf{n}$ & $\%$ & $\mathbf{n}$ & $\%$ & $\mathbf{n}$ & $\%$ \\
\hline Student & 12 & 54.5 & 8 & 57.2 & 5 & 55.6 & 3 & 50 & 28 & 55.0 \\
\hline $\begin{array}{l}\text { Retail merchant } \\
\text { lemployee/ worker }\end{array}$ & 5 & 22.6 & 1 & 7.1 & 1 & 11.1 & 0 & 0 & 7 & 13.7 \\
\hline Self-employed/ manager/director & 2 & 9.2 & 2 & 14.3 & 0 & 0 & 0 & 0 & 4 & 7.8 \\
\hline
\end{tabular}




\begin{tabular}{|c|c|c|c|c|c|c|c|c|c|c|}
\hline Housewife & 1 & 4.5 & 2 & 14.3 & 1 & 11.1 & 0 & 0 & 4 & 7.8 \\
\hline Unemployed & 2 & 9.5 & 1 & 7.1 & 2 & 22.2 & 3 & 50 & 8 & 15.7 \\
\hline \multicolumn{11}{|l|}{ Marital status } \\
\hline Unmarried/engaged & $20 / 5$ & 90.9 & $12 / 2$ & 85.7 & $7 / 2$ & 77.8 & $6 / 3$ & 100 & $45 / 12$ & 88.2 \\
\hline Married & 2 & 9.1 & 2 & 14.3 & 2 & 22.2 & 0 & 0 & 6 & 11.8 \\
\hline Divorced & 0 & 0 & 0 & 0 & 0 & 0 & 0 & 0 & 0 & 0 \\
\hline Children & $\mathbf{n}$ & $\%$ & $\mathbf{n}$ & $\%$ & $\mathbf{n}$ & $\%$ & $\mathbf{n}$ & $\%$ & $\mathbf{n}$ & $\%$ \\
\hline No & 21 & 95.5 & 12 & 85.7 & 8 & 88.9 & 6 & 100 & 47 & 92.2 \\
\hline Yes & 1 & 4.5 & 2 & 14.3 & 1 & 11.1 & 0 & 0 & 4 & 7.8 \\
\hline \multicolumn{11}{|l|}{ Offsetting Behaviours } \\
\hline No & 8 & 36.4 & 0 & 0 & 9 & 100 & 2 & 33.3 & 19 & 37.3 \\
\hline Vomiting & 2 & 9.1 & 12 & 85.7 & 0 & 0 & 3 & 50 & 17 & 33.4 \\
\hline $\begin{array}{l}\text { Laxatives/diuretics } \\
\text { /physical hyperactivity }\end{array}$ & 9 & 40.9 & 0 & 0 & 0 & 0 & 1 & 6.7 & 10 & 19.6 \\
\hline Vomiting+others & 3 & 13.6 & 2 & 14.3 & 0 & 0 & 0 & 0 & 5 & 9.7 \\
\hline Smoking & $\mathbf{n}$ & $\%$ & $\mathbf{n}$ & $\%$ & $\mathbf{n}$ & $\%$ & $\mathbf{n}$ & $\%$ & $\mathbf{n}$ & $\%$ \\
\hline No & 18 & 81.8 & 6 & 42.9 & 2 & 22.2 & 5 & 83.3 & 31 & 60.8 \\
\hline$<10 /$ day & 3 & 13.6 & 7 & 50 & 4 & 44.4 & 1 & 16.7 & 15 & 29.4 \\
\hline$>10 /$ day & 1 & 4.5 & 1 & 7.1 & 3 & 33.3 & 0 & 0 & 5 & 9.8 \\
\hline Menstrual cycle & $\mathbf{n}$ & $\%$ & $\mathbf{n}$ & $\%$ & $\mathbf{n}$ & $\%$ & $\mathbf{n}$ & $\%$ & $\mathbf{n}$ & $\%$ \\
\hline Amenorrea* & 22 & 100 & 1 & 7.1 & 1 & 11.1 & 2 & 33.3 & 26 & 51.0 \\
\hline Regular & 0 & 0 & 13 & 92.9 & 8 & 88.9 & 4 & 6.7 & 25 & 49.0 \\
\hline \multicolumn{11}{|l|}{ Calories prescribed at admission } \\
\hline $1300-1500$ & 10 & 45.5 & 4 & 28.6 & 0 & 0 & 3 & 50 & 17 & 33.3 \\
\hline $1500-1700$ & 9 & 40.9 & 10 & 71.4 & 3 & 33.3 & 3 & 50 & 25 & 49.0 \\
\hline $1700-1900$ & 1 & 4.5 & 0 & 0 & 6 & 66.7 & 0 & 0 & 7 & 13.7 \\
\hline $1900-2100$ & 2 & 9.1 & 0 & 0 & 0 & 0 & 0 & 0 & 2 & 4.0 \\
\hline Calories prescribed at discharge & $\mathbf{n}$ & $\%$ & $\mathbf{n}$ & $\%$ & $\mathbf{n}$ & $\%$ & $\mathbf{n}$ & $\%$ & $\mathbf{n}$ & $\%$ \\
\hline $1300-1500$ & 0 & 0 & 2 & 14.3 & 0 & 0 & 0 & 0 & 2 & 4.0 \\
\hline $1500-1700$ & 3 & 13.6 & 12 & 85.7 & 3 & 33.3 & 4 & 66.7 & 22 & 43.1 \\
\hline $1700-1900$ & 12 & 54.5 & 0 & 0 & 6 & 66.7 & 2 & 33.3 & 20 & 39.2 \\
\hline $1900-2100$ & 7 & 31.8 & 0 & 0 & 0 & 0 & 0 & 0 & 7 & 13.7 \\
\hline Liver Ultrasound at admission & $\mathbf{n}$ & $\%$ & $\mathbf{n}$ & $\%$ & $\mathbf{n}$ & $\%$ & $\mathbf{n}$ & $\%$ & $\mathbf{n}$ & $\%$ \\
\hline Normal & 21 & 95.5 & 13 & 72.9 & 4 & 44.4 & 6 & 100 & 44 & 86.3 \\
\hline I grade Steatosis & 1 & 4.5 & 1 & 7.1 & 1 & 11.1 & 0 & 0 & 3 & 5.9 \\
\hline II grade Steatosis & 0 & 0 & 0 & 0 & 4 & 44.4 & 0 & 0 & 4 & 7.8 \\
\hline Liver Ultrasound at discharge & $\mathbf{n}$ & $\%$ & $\mathbf{n}$ & $\%$ & $\mathbf{n}$ & $\%$ & $\mathbf{n}$ & $\%$ & $\mathbf{n}$ & $\%$ \\
\hline Normal & 21 & 95.5 & 13 & 72.9 & 4 & 44.4 & 6 & 100 & 44 & 86.3 \\
\hline I grade Steatosis & 1 & 4.5 & 1 & 7.1 & 4 & 44.4 & 0 & 0 & 6 & 11.8 \\
\hline II grade Steatosis & 0 & 0 & 0 & 0 & 1 & 11.1 & 0 & 0 & 1 & 1.9 \\
\hline
\end{tabular}


EDNOS=Eating Disorder not otherwise specified

*14 patients (12 AN, 2 EDNOS) recovered menstruations

**8 AN and 1 EDNOS received ONS (300 kcal/day)

In details, Table 2 highlights a significant and progressive weight gain in AN patients both during the residential stage and at the following FU. BMI and FM significantly increased since the first month, whilst FFM (Fat Free Mass) and TBW (Total Body Water) later.

At entry, $45.5 \%$ AN patients received a $1300-1500 \mathrm{Kcal} /$ day diet; eight patients (36.4\%) received oral nutritional supplements (ONS) accounting for an additional calorie amount of about $300 \mathrm{kcal} /$ day. Resting Energy Expenditure (REE) progressively increased, reaching significance at $5^{\text {th }}$ month. Menstruation spontaneously occurred in 12 patients, after an average of 17 (5-48) months amenorrhea and without estro-progestin prescription. As regards haemato-biochemical data, there were no significant differences at entry, $1-3-5^{\text {th }}$ month and at FU observation (data not shown).

At discharge, dietary regimen was $1700-1900 \mathrm{Kcal} /$ day without ONS integration in 12 patients $(54.5 \%$ of the whole group).

Table 2: Body composition and Resting Energy Expenditure changes in 22 patients with Anorexia Nervosa.

\begin{tabular}{|c|c|c|c|c|c|}
\hline & At Entry & 1 month & 3 months & 5 months & Follow Up +++ \\
\hline \multirow[t]{2}{*}{ Weight (kg) } & $37.5 \pm 4.4$ & $38.9 \pm 4.2$ * & $42.3 \pm 3.9$ * & $44.7 \pm 4.2$ * & $46 \pm 5$ * \\
\hline & $(30.9-46.0)$ & $\Delta 1.4 \pm 0.2$ & $\Delta 4.8 \pm 0.5$ & $\Delta 7.2 \pm 0.2$ & $\Delta 8.5 \pm 0.6$ \\
\hline BMI & $15.2 \pm 1.5$ & $15.8 \pm 1.5^{*}$ & $17.1 \pm 1.3$ * & $18.1 \pm 1.5$ & $18.6 \pm 1.6^{*}$ \\
\hline$\left(\mathrm{kg} / \mathrm{m}^{2}\right)$ & $(12.8-18.3)$ & $\Delta 0.6 \pm 0$ & $\Delta 1.9 \pm 0.2$ & $\Delta 0.6 \pm 0$ & $\Delta 3.4 \pm 0.1$ \\
\hline FFM & $35.1 \pm 3.1$ & $34.8 \pm 3.6$ & $36.7 \pm 3.7 \Delta 1.6 \pm 0.6$ & $37.9 \pm 4.2^{* * *}$ & $37.1 \pm 4.4^{* * *}$ \\
\hline (kg) & $(30.9-42.0)$ & $\Delta-0.3 \pm 0.5$ & & $\Delta 2.8 \pm 1.1$ & $\Delta 2 \pm 1.3$ \\
\hline FFM & $91 \pm 6.7$ & $87 \pm 6.2^{* *}$ & $85.3 \pm 5.9^{* *}$ & $83.6 \pm 6.2^{* * *}$ & $80.8 \pm 4.9^{* *}$ \\
\hline$(\%)$ & $(76.4-100)$ & $\Delta-4 \pm 0.5$ & $\Delta-5.7 \pm 0.8$ & $\Delta-7.4 \pm 0.5$ & $\Delta-10.2 \pm 1.8$ \\
\hline FM & $3.7 \pm 2.9$ & $5.3 \pm 2.6 \mathrm{~kg}$ ** & $6.4 \pm 2.6^{*}$ & $7.5 \pm 2.8^{*}$ & $8.8 \pm 2.3^{*} \Delta 5.1 \pm 0.6$ \\
\hline (kg) & $(0-8)$ & $\Delta 1.6 \pm 0.3$ & $\Delta 2.7 \pm 0.3$ & $\Delta 3.8 \pm 0.1$ & \\
\hline FM & $9.8 \pm 8.7$ & $12.9 \pm 6.3 \%$ & $14.7 \pm 5.9$ *** & $16.4 \pm 6.2^{* * *}$ & $19.2 \pm 4.9^{* *}$ \\
\hline$(\%)$ & $(0-35.7)$ & $\Delta 3.1 \pm 2.4$ & $\Delta 4.9 \pm 2.8$ & $\Delta 6.6 \pm 2.5$ & $\Delta 9.4 \pm 3.8$ \\
\hline TBW & $25.9 \pm 2.3$ & $25.5 \pm 2.8 \mathrm{lt}$ & $27 \pm 2.7$ It & $27.9 \pm 3.1 \mathrm{lt}$ & $27.2 \pm 3.1 \mathrm{lt}$ \\
\hline \multirow[t]{2}{*}{ (It) } & $(21.4-30.6)$ & $\Delta-0.4 \pm 0.5$ & $\Delta 1.1 \pm 0.4$ & $* * *$ & $\Delta 1.3 \pm 0.8$ \\
\hline & & & & $\Delta 2 \pm 0.8$ & \\
\hline TBW & $67.1 \pm 4.7$ & $63.5 \pm 4.5^{* * *}$ & $62.7 \pm 4.3^{* *}$ & $61.5 \pm 4.5^{* *}$ & $59.3 \pm 3.6$ * \\
\hline$(\%)$ & $(56.7-73.0)$ & $\Delta-3.6 \pm 0.2$ & $\Delta-4.4 \pm 0.4$ & $\Delta-5.6 \pm 0.2$ & $\Delta-7.8 \pm 1.1$ \\
\hline \multirow[t]{2}{*}{ REE (kcal) } & $941 \pm 246$ & $982 \pm 237$ & $1013 \pm 137$ & $1129 \pm 156^{\star * *}$ & $1090 \pm 149$ \\
\hline & $(550-1373)$ & $\Delta 41 \pm 9$ & $\Delta 72 \pm 109$ & $\Delta 188 \pm 90$ & $\Delta 149 \pm 97$ \\
\hline $\mathbf{R Q}$ & $0.9 \pm 0.1$ & $0.89 \pm 0.1$ & $0.94 \pm 0.01$ & $0.95 \pm 0.01$ & $0.93 \pm 0.1$ \\
\hline & $(0.63-1.38)$ & & & & \\
\hline REE/FFM & $26.6 \pm 6.1$ & $27.7 \pm 5.7$ & $27.9 \pm 4.6$ & $29.5 \pm 5.7$ & $29.6 \pm 4.7$ \\
\hline $\mathrm{Kcal} / \mathrm{Kg}$ & & & & & \\
\hline
\end{tabular}

+++Follow Up 1 month after discharge

Values are expressed as mean \pm Standard Deviation and range values (first column);

$\Delta$ expresses the difference compared to the basal values;

${ }^{*} p<0.001 ; *^{*} p<0.01 ; * * * p<0.05$ express the significance compared to the basal values.

FFM=Fat Free Mass; FM=Fat Mass; TBW=Total Body water; REE=Resting Energy Expenditure; RQ=Respiratory Quotient;
Body weight remained substantially stable in the $14 \mathrm{BN}$ patients (Table 3 ), with a slight decrease at the $1^{\text {st }}$ month. Haemato-biochemical exams were normal and stable all over the time of observation (data not shown), as well as BP and $H R$, whilst REE slightly decreased after 3 months. Dietetic prescription remained substantially unchanged for BN patients, at the Residence and at discharge, with a mean energy intake of 1500-1700 kcal/day.

Table 3: Body composition and Resting Energy Expenditure changes in 14 patients with Bulimia Nervosa. 


\begin{tabular}{|c|c|c|c|c|c|}
\hline & At Entry & 1 month & 3 months & 5 months & $\begin{array}{l}\text { Follow Up } \\
+++\end{array}$ \\
\hline Weight (kg) & $\begin{array}{l}66.2 \pm 16.5 \\
(49.2-102)\end{array}$ & $65.2 \pm 15.6^{* * *}$ & $64.7 \pm 14.9$ & $64.5 \pm 14$ & $65.1 \pm 15.5$ \\
\hline BMI $\left(\mathrm{kg} / \mathrm{m}^{2}\right)$ & $\begin{array}{l}25.4 \pm 6.8 \\
(18.5-43)\end{array}$ & $25 \pm 6.5^{* * *}$ & $24.7 \pm 6.3$ & $24.7 \pm 6$ & $24.8 \pm 6.7$ \\
\hline FFM (kg) & $\begin{array}{l}44.3 \pm 4.5 \\
(38-52.6)\end{array}$ & $43.9 \pm 3.6$ & $44.6 \pm 3.6$ & $44.3 \pm 3.6$ & $44.7 \pm 3.6$ \\
\hline FFM (\%) & $\begin{array}{l}69.2 \pm 10.5 \\
(47-84.7)\end{array}$ & $69.6 \pm 10.6$ & $70.8 \pm 9.7$ & $70.3 \pm 8.7$ & $70.7 \pm 9.4$ \\
\hline FM (kg) & $\begin{array}{l}21.8 \pm 13 \\
(7.5-54.1)\end{array}$ & $21.3 \pm 13$ & $\begin{array}{l}20.1 \pm 11.7 \\
\Delta-1.1\end{array}$ & $20.2 \pm 10.7$ & $20.3 \pm 12.8$ \\
\hline FM (\%) & $\begin{array}{l}30.7 \pm 10.5 \\
(15.3-53)\end{array}$ & $30.4 \pm 10.6$ & $29.1 \pm 9.7$ & $29.7 \pm 9.7$ & $29.2 \pm 9.4$ \\
\hline TBW (It) & $\begin{array}{l}32.1 \pm 3.2 \\
(27.8-37.5)\end{array}$ & $31.9 \pm 2.7$ & $32.3 \pm 2.9$ & $31.8 \pm 2.8$ & $32.4 \pm 3$ \\
\hline TBW ( \%) & $\begin{array}{l}50.1 \pm 7.7 \\
(35.6-61.9)\end{array}$ & $50.6 \pm 7.7$ & $\begin{array}{l}51.2 \pm 7.1 \\
\star * \star\end{array}$ & $50.6 \pm 6.6$ & $51.2 \pm 6.6$ \\
\hline REE (kcal) & $\begin{array}{l}1363 \pm 160 \\
(1051-1757)\end{array}$ & $1292 \pm 192$ & $\begin{array}{l}1158 \pm 202 \\
\star \star *\end{array}$ & $1288 \pm 264$ & $1284 \pm 252$ \\
\hline $\mathbf{R Q}$ & $\begin{array}{l}0.85 \pm 0.1 \\
(0,54-1.6)\end{array}$ & $0.91 \pm 0.1$ & $0.92 \pm 0.1$ & $0.9 \pm 0.1$ & $0.87 \pm 0.1$ \\
\hline REE /FFM (kcal/kg) & $30.7 \pm 4.4$ & $29.2 \pm 3.5$ & $\begin{array}{l}25.6 \pm 3.2 \\
* *\end{array}$ & $29 \pm 5$ & $28.6 \pm 4.8$ \\
\hline
\end{tabular}

+++ Follow Up 1 month after discharge.

Values are expressed as mean \pm Standard Deviation and range values (at entry only) (in the first column);

${ }^{*} p<0.001 ; * * p<0.01 ; * * * p<0.05$ express the significance compared to the basal values.

FFM=Fat Free Mass; FM=Fat Mass; TBW=Total Body water; REE=Resting Energy Expenditure; RQ=Respiratory Quotient;

Table 4 describes a significant and gradual weight loss in 9 BED patients, both during the residential period and at the first FU. BIA exam showed a progressive FM loss, both in percent and absolute values, during the therapeutic residential period and at the first FU.

REE progressively and significantly decreased after 3 months and at the first FU. Main dietary regimen prescribed to BED patients was of about $1700-1900 \mathrm{Kcal}$, and remained unchanged at the discharge. Of interest, in 3/4 BED patients, liver steatosis ameliorated, from grade 2 (moderate) to grade 1 (slight). Haemato-biochemical exams, as well as systolic and diastolic BP remained substantially unchanged ( data not shown ).

Table 4: Body composition and Resting Energy Expenditure changes in 9 patients with Binge Eating Disorders.

\begin{tabular}{|c|c|c|c|c|c|}
\hline At Entry & 1 month & 3 months & 5 months & Follow Up +++ & At Entry \\
\hline \multirow{2}{*}{ Weight (kg) } & $122.5 \pm 25$ & \multirow{2}{*}{$118.5 \pm 24^{*}$} & \multirow{2}{*}{$111.5 \pm 22.2^{*}$} & \multirow{2}{*}{$106.9 \pm 21.3^{*}$} & \multirow{2}{*}{$106 \pm 21.8^{*}$} \\
\hline & $(85.9-181.3)$ & & & & \\
\hline \multirow{2}{*}{ BMI $\left(\mathbf{k g} / \mathbf{m}^{2}\right)$} & $45.3 \pm 9.4$ & \multirow{2}{*}{$43.9 \pm 9^{*}$} & \multirow{2}{*}{$41.4 \pm 8.6^{*}$} & $39.7 \pm 8.4^{*}$ & $39.3 \pm 8.5^{\star}$ \\
\hline & $(30.9-63.5)$ & & & & \\
\hline \multirow{2}{*}{ FFM (kg) } & $58.5 \pm 6.4$ & \multirow{2}{*}{$57.6 \pm 6.1$} & \multirow{2}{*}{$55.6 \pm 5.6^{* *}$} & $56.3 \pm 5.3$ & $55.2 \pm 6.2$ \\
\hline & $(47.0-71.0)$ & & & & \\
\hline FFM (\%) & $48.6 \pm 6.1$ & $49.4 \pm 5.7^{\star \star \star}$ & $50.7 \pm 6.3^{*}$ & $53.7 \pm 6.6^{*}$ & $53.2 \pm 8.3^{\star * *}$ \\
\hline
\end{tabular}




\begin{tabular}{|c|c|c|c|c|c|}
\hline & $(39.0-39.7)$ & & & & \\
\hline \multirow{2}{*}{ FM (kg) } & $63.9 \pm 20$ & \multirow{2}{*}{$60 \pm 18.9^{*}$} & \multirow{2}{*}{$55.9 \pm 17.2^{*}$} & $50.6 \pm 17.3^{*}$ & $50.9 \pm 19^{* *}$ \\
\hline & $(36.0-110.0)$ & & & & \\
\hline \multirow{2}{*}{ FM (\%) } & $51.4 \pm 6.1$ & \multirow{2}{*}{$50.5 \pm 5.7^{* * *}$} & \multirow{2}{*}{$49.2 \pm 6.3^{*}$} & $46.3 \pm 6.6^{*}$ & $46.7 \pm 8.3^{\star * *}$ \\
\hline & $(40.3-60.9)$ & & & & \\
\hline \multirow{2}{*}{ TBW (It) } & $43.1 \pm 5.2$ & \multirow{2}{*}{$42 \pm 5.7$} & \multirow{2}{*}{$40.9 \pm 5.0^{* *}$} & $41.4 \pm 4.3^{*}$ & $41.4 \pm 5.0$ \\
\hline & $(34.7-54.0)$ & & & & \\
\hline \multirow{2}{*}{ TBW ( \%) } & $35.7 \pm 3.6$ & \multirow{2}{*}{$35.9 \pm 3.4$} & \multirow{2}{*}{$37.3 \pm 4.4^{\star * *}$} & $39.4 \pm 4.3^{*}$ & $39.6 \pm 4.8^{* *}$ \\
\hline & $(29.9-42.0)$ & & & & \\
\hline \multirow{2}{*}{ REE (kcal) } & $1960 \pm 396$ & \multirow{2}{*}{$1672 \pm 231$} & \multirow{2}{*}{$1584 \pm 315^{\star \star *}$} & $1806 \pm 376$ & $1669 \pm 367^{\star \star \star}$ \\
\hline & $(1439-2511)$ & & & & \\
\hline \multirow{2}{*}{$\mathbf{R Q}$} & $0.95 \pm 0.05$ & \multirow{2}{*}{$0.92 \pm 0.03$} & \multirow{2}{*}{$0.95 \pm 0.04$} & $0.94 \pm 0.1$ & $0.91 \pm 0.05$ \\
\hline & $(0.75-1.0)$ & & & & \\
\hline
\end{tabular}

+++ Follow Up 1 month after discharge

Values are expresses as mean \pm Standard Deviation and range values (in the first column);

FFM=Fat Free Mass; FM=Fat Mass; TBW=Total Body water; REE=Resting Energy Expenditure; $R Q=$ Respiratory Quotient;

$*_{p}<0.001 ; * * p<0.01 ; * * * p<0.05$ express the significance compared to the basal values.

Table 5 shows no significant clinical change for EDNOS patients, possibly due to the wide variability of symptoms gathered in this definition and the small number of patients evaluated. Nonetheless, when considering separately the 4 EDNOS patients with $B M \mathrm{~K}<18.5 \mathrm{~kg} / \mathrm{m}^{2}$, body weight and $\mathrm{FM}$ gain was observed at the $3^{\text {rd }}$ and $5^{\text {th }}$ month of the residential stage.

Three of these 4 patients had amenorrhea at entry; 2 of them recovered menstruation after a mean period of $9 \pm 5$ months. EDNOS patients received a dietetic regimen of $1300-1700 \mathrm{kcal} /$ day. At the discharge all patients received a dietetic prescription of about $1500-1700 \mathrm{kcal} /$ day.

Table 5: Body composition and REE changes in 6 EDNOS patients.

\begin{tabular}{|c|c|c|c|c|c|}
\hline & \multirow{2}{*}{ AT ENTRY } & \multirow{2}{*}{1 MONTH } & \multirow{2}{*}{3 MONTHS } & \multirow{2}{*}{5 MONTHS } & 1 Follow Up \\
\hline & & & & & (6 months) \\
\hline \multirow{2}{*}{ Weight (kg) } & $51.3 \pm 13.6$ & \multirow{2}{*}{$51.2 \pm 12.4$} & \multirow{2}{*}{$50.7 \pm 10$} & \multirow{2}{*}{$51 \pm 5.8$} & \multirow{2}{*}{$55.8 \pm 10.2$} \\
\hline & $(36.8-74.3)$ & & & & \\
\hline \multirow{2}{*}{ BMI $\left(\mathrm{kg} / \mathrm{m}^{2}\right)$} & $20.5 \pm 4.9$ & \multirow{2}{*}{$20.2 \pm 5.5$} & \multirow{2}{*}{$20.8 \pm 4.6$} & \multirow{2}{*}{$19.7 \pm 1.3$} & \multirow{2}{*}{$22 \pm 4.8$} \\
\hline & $(14.7-31.7)$ & & & & \\
\hline \multirow{2}{*}{ FFM (kg) } & $40.5 \pm 5.3$ & \multirow{2}{*}{$39.5 \pm 5.3$} & \multirow{2}{*}{$40 \pm 4.8$} & \multirow{2}{*}{$41 \pm 3.5$} & \multirow{2}{*}{$41.6 \pm 2$} \\
\hline & $(32.4-45.3)$ & & & & \\
\hline \multirow{2}{*}{ FFM (\%) } & $81.3 \pm 12.3$ & \multirow{2}{*}{$78.8 \pm 11$} & \multirow{2}{*}{$77 \pm 9.3$} & \multirow{2}{*}{$80.6 \pm 3.8^{* * *}$} & \multirow{2}{*}{$76 \pm 10$} \\
\hline & $(58.8-95.0)$ & & & & \\
\hline \multirow{2}{*}{ FM (kg) } & $11 \pm 10$ & \multirow{2}{*}{$11.7 \pm 9.1$} & \multirow{2}{*}{$12.7 \pm 7.6$} & \multirow{2}{*}{$10 \pm 3^{* * *}$} & \multirow{2}{*}{$14.3 \pm 8.7$} \\
\hline & $(2.2-30.6)$ & & & & \\
\hline \multirow{2}{*}{ FM (\%) } & $18.7 \pm 12.2$ & \multirow{2}{*}{$21.1 \pm 10.6$} & \multirow{2}{*}{$22.9 \pm 9.3$} & \multirow{2}{*}{$19.4 \pm 3.8^{* * *}$} & \multirow{2}{*}{$24 \pm 10$} \\
\hline & $(5.0-41.2)$ & & & & \\
\hline \multirow{2}{*}{ TBW (It) } & $29.4 \pm 4.4$ & \multirow{2}{*}{$28.7 \pm 3.8$} & \multirow{2}{*}{$29 \pm 3.1$} & \multirow{2}{*}{$30 \pm 2.5$} & \\
\hline & $(22.2-33.0)$ & & & & $30 . \angle \pm 1.8$ \\
\hline
\end{tabular}




\begin{tabular}{|c|c|c|c|c|c|}
\hline \multirow{2}{*}{ TBW ( \%) } & $58.8 \pm 8.5$ & \multirow{2}{*}{$57.5 \pm 7.7$} & \multirow{2}{*}{$56 \pm 7^{* * *}$} & \multirow{2}{*}{$58.9 \pm 3.5$} & \multirow{2}{*}{$55 \pm 7.5$} \\
\hline & $(43.6-69.6)$ & & & & \\
\hline \multirow{2}{*}{ REE (kcal) } & $1122 \pm 333$ & \multirow{2}{*}{ ns } & \multirow{2}{*}{ ns } & \multirow{2}{*}{ ns } & \multirow{2}{*}{ ns } \\
\hline & $(802-1540)$ & & & & \\
\hline \multirow{2}{*}{$\mathbf{R Q}$} & $0.88 \pm 0.01$ & \multirow{2}{*}{ ns } & \multirow{2}{*}{ ns } & \multirow{2}{*}{ ns } & \multirow{2}{*}{ ns } \\
\hline & $(0.82-1.7)$ & & & & \\
\hline
\end{tabular}

Values are expresses as mean \pm Standard Deviation and range values (in the first column);

${ }^{*} p<0.001 ; *^{*} p<0.01 ; * * * p<0.05$ express the significance compared to the basal values;

$\mathrm{Ns}=$ not significant variations versus at admission values.

FFM=Fat Free Mass; FM=Fat Mass; TBW=Total Body water; $\mathrm{REE}=$ Resting Energy Expenditure; $\mathrm{RQ}=$ Respiratory Quotient;

\section{Discussion}

The study retrospectively describes a clinical nutritional experience in a Residential Centre for Eating Disorders, unique in Southern Italy, and has the objective to collect information on short term clinical and nutritional outcome of 51 out 71 patients affected by EDs, AN being the most frequent. This Residential Centre for Eating Disorders mostly hosts female patients, in particular AN with a $\mathrm{BMI}>13 \mathrm{~kg} / \mathrm{m}^{2}$. In the Residence, patients follow a strictly supervised dietary regimen plus several rehabilitative activities.

As emerged from our patients characteristics, EDs involve a wide age range, from early adolescence to the adult age. As a matter of fact, age range of the patients admitted at the rehabilitative program was $13-40$ years with disease duration of 1-24 years. Twenty ( $4 \mathrm{~m}, 16 \mathrm{f}$ ) patients have been excluded because not attending the follow up at the first month. These patients did not differ as far as age and socio-demographic characteristics from the patients described in the study. Disease length was longer (8.5 \pm 5.6 years) for BN and BED than AN ( $5.9 \pm 4.3$ years) patients. This finding could be due to a poor awareness for BN by family, society and public health operators than in the case of AN. Patients birthplace reflects the location of Center. The Center is in Basilicata region, is a referring center and unique in Southern Italy.

From the analysis of patients' socio-cultural level, it is confirmed that these patients belong not only to high social level classes, $61 \%$ patients coming from medium-low social classes.

The restoration of a healthy eating behavior, according to patients' need, and careful clinical supervision succeeded in reducing and often interrupting binge eating and purging behaviors.

As regards the clinical nutritional view, we underscore the gradual weight gain in AN patients, with no refeeding syndrome episodes, the improvement of body composition parameters at discharge and at the FU, with a mean 200 $\mathrm{kcal} /$ day REE increase at $5^{\text {th }}$ month observation and the restoration of menstruation in 12 patients after a mean length of amenorrhea of 17 months ( $\min 5-\max 48$ ).

In AN patients, weight recovery, accompanied by FM, FFM and TBW restoration, has certainly favored restoration of the menstrual cycle. This finding is also explained, among others, by the described increased leptin production by the adipose tissue, at least partially recovered.

BN patients did not have baseline severe clinical and biochemical abnormalities, had a normal body weight, remained substantially stable with a slight final decrease of FM and TBW. Even if not statistically significant, we consider these decreases as a possible effect of adopting regular food behaviors, with the elimination of binge or purging conducts. In fact, no vomiting episodes during the stage were recorded.

The strict cooperation and integration of several professional figures in a specialized team, as suggested by the American Psychiatric Association (APA) Guidelines, seems mandatory for the treatment of ED as confirmed in our experience, in particular to avoid/face nutritional complications such as the refeeding syndrome, educate the patient to an healthy diet and behaviors, help to consider beliefs, motivations, conflicts and dysfunctional feelings linked to ED and finally to reduce and progressively eliminate binge eating and purging behaviors. The specialized team also supports the family and works to prevent relapse at home.

In order to prepare patients (and family) to return to their family and social environment in the last two years, among other interventions, The Assisted Family Nutrition Therapy (AFNT) was introduced. AFNT starts from the $45^{\text {th }}$ day of residency, after the first snacks consumed with parents and a series of informative/training meetings. An ad hoc buffet is set up in the Residence which is made up of dishes similar to those supplied during the Nutritional Therapy trays ( $1^{\text {st }}$ course, $2^{\text {nd }}$ course, side dish, bread and fruit). The family is united to eat, often for the first time after months of separation!

Nutritional Rehabilitation (NR), which is the main aim of the present study, has no primary weight objectives and is focused on a gradual normalization of food behavior and education to appropriate food choices, by considering individual energy requirements with the progressive and agreed reintroduction of initially refused foods, going out from the dichotomy "allowed-forbidden foods" with the recovery of taste and physiological signals of appetite and satiety. 
The safety and the beneficial effects of the nutritional intervention, in particular in AN patients, is also confirmed by the increase of REE with the harmonious increase of both FFM and FM: the occurrence of menstruation, without pharmacological intervention, as well as the amelioration of some haemato-biochemical and clinical parameters such as increased blood pressure values (data not shown).

Finally, in AN patients, Nutritional Rehabilitation avoided the onset of the refeeding syndrome. For these patients recommended food intake gradually increased to an isocaloric diet (1700-1900 kcal/day) without the prescription of liquid ONS at discharge.

In BED patients, the prescribed diet is only mildly hypocaloric (in 67\% between 1700-1900 Kcal/day), without qualitative and quantitative reductions, complete and varied, in contrast with the frequently unsuccessful too restrictive prescriptions usually characterizing the case histories of these patients which could also contribute to the endurance of the disease.

This study has some limitations. It is mono-centric; sample size is rather small; patient's selection excluded severe cases of eating disorders diseases, long term follow-up observations are not yet available. On the other hand, quite unexpected, not many data are available on the safety and clinical nutritional outcome of residential rehabilitative programs for the treatment of eating disorders. In this study we have mostly focused our attention on clinical nutritional outcome: it is noteworthy to record that weight gain/loss characteristics were optimal, without refeeding syndrome, abnormal FM increase or FFM loss respectively. Further observations are necessary and multicentre case-control studies are needed to confirm our short term observations and to verify the effectiveness in the long term of this therapeutic procedure.

\section{Conclusion}

In conclusion despite data in literature are lacking and the study group is quite small, we can confirm the safety, at least in the short term, of a residential approach for EDs, without side effects, a reasonable adherence to therapeutic protocol, with satisfying clinical nutritional improvements.

\section{References}

1. Treasure J, Claudino AM, Zucker N (2010) Eating disorders. Lancet 375: 583-593.

2. Berkman ND, Lohr KN, Bulik CM (2007) Outcomes of eating disorders: a systematic review of the literature. Int J Eat Disord 40: 293-309.

3. Brewerton TD, Costin C (2011) Long-term outcome of residential treatment for anorexia nervosa and bulimia nervosa. Eat Disord 19: 132-144.

4. Weltzin T, Bean P, Klosterman E, Lee HJ, Welk-Richards R (2014) Sex differences in the effects of residential treatment on the quality of life of eating disorder patients. Eat Weight Disord 20: 301-310.
5. Beumont PJV (2002) Clinical presentation of anorexia and bulimia nervosa. In : Brownell KD, Fairburn CG, eds. Eating disorders and obesity: a comprehensive handbook 2nd edn. New York: Guilford.

6. Lask B, Bryant-Waugh R (1993) Childhood onset anorexia nervosa and related eating disorders. In: Howe: Lawrence Erlbaum.

7. Beumont $P$, Hay $P$, Beumont $D$, Birmingham L, Derham $H$, et al. (2004) Royal Australian and New Zealand College of Psychiatrists Clinical Practice Guidelines Team for Anorexia Nervosa. Australian and New Zealand clinical practice guidelines for the treatment of anorexia nervosa. Aust $\mathrm{N} \mathrm{Z} \mathrm{J} \mathrm{Psychiatry} \mathrm{38:}$ 659-670.

8. Frisch MJ, Herzog DB, Franko DL (2006) Residential treatment for eating disorders. Int J Eat Disord 39: 434-442.

9. American Psychiatric Association (2006) Practice guidelines for the treatment of patients with eating disorders. Practice guidelines for the treatment of psychiatric disorders.3rd edn. Arlington: American Psychiatric Association pp: 1097-1222.

10. Williams PM, Goodie J, Motsinger CD (2008) Treating eating disorders in primary care. Am Fam Physician 77: 187-195.

11. Reiter CS, Graves L (2010) Nutrition therapy for eating disorders. Nutr Clin Pract 25: 122-136.

12. National Collaborating Centre for Mental Health (2004) National Clinical Practice Guideline: Eating Disorders: core interventions in the treatment and management of Anorexia Nervosa, Bulimia Nervosa, and related eating disorders. National Istitute for Clinical Excellence.

13. Crow SJ, Mitchell JE, Roerig JD, Steffen K (2009) What potential role is there for medication treatment in anorexia nervosa? Int J Eat Disord 42: 1-8.

14. Shapiro JR, Berkman ND, Brownley KA, Sedway JA, Lohr KN, et al. (2007) Bulimia nervosa treatment: a systematic review of randomized controlled trials. Int J Eat Disord 40: 321-336.

15. Hay PJ, Bacaltchuk J (2008) Bulimia nervosa. BMJ Clin Evid pii: 1009.

16. Mitchell JE, Agras S, Wonderlich S (2007) Treatment of bulimia nervosa: where are we and where are we going? Int J Eat Disord 40: 95-101.

17. Halmi KA (2009) Salient components of a comprehensive service for eating disorders. World Psychiatry 8: 150-155.

18. Tonkin RS (1997) Evaluation of a summer camp for adolescents with eating disorders. J Adolesc Health 20: 412-413.

19. Bean P, Weltzin T (2006) Evolution of symptom severity during residential treatment of females with eating disorders. Eat Weight Disord 6: 197-204.

20. Fittig $E$, Jacobi $C$, Backmund $H$, Gerlinghoff $M$, Wittchen $H U$ (2008) Effectiveness of day hospital treatment for anorexia nervosa and bulimia nervosa. Eur Eat Disord Rev 16: 341-351.

21. Naab S, Schlegl S, Korte A, Heuser J, Fumi M, et al. (2013) Effectiveness of a multimodal inpatient treatment for adolescents with anorexia nervosa in comparison with adults: an analysis of a specialized inpatient setting : treatment of adolescent and adult anorexics. Eat Weight Disord 18: 167-173.

22. DSM IV-TR (2000) Diagnostic and Statistical Manual of Mental Disorders, American Psychiatric Association. 
23. DSM-5 (2014) Diagnostic and Statistical Manual of Mental Disorders. American Psychiatric Association.
24. Saverymuttu SH, Joseph AE, Maxwell JD (1986) Ultrasound scanning in the detection of hepatic fibrosis and steatosis. $\mathrm{Br}$ Med J Clin Res Ed 292: 13-15. 